\title{
Backscattered Electron Imaging with an EBSD Detector
}

\section{Robert A. Schwarzer ${ }^{1 *}$ and Jarle Hjelen ${ }^{2}$}

${ }^{1}$ Kappstr. 65, D-71083 Herrenberg, Germany

${ }^{2}$ Dept. Mat. Sci. Eng. NTNU, Alfred Getz vei 2, NO-7491 Trondheim, Norway

*schwarzer@ebsd.info

\section{Introduction}

In the scanning electron microscope (SEM), backscattered electrons (BSEs) can be used as a complement to secondary electrons (SEs) for imaging bulk sample surfaces. If the sample is steeply tilted, as is the case in electron backscatter diffraction (EBSD) measurements, the usual backscattered electron detectors above the sample are ineffective. The EBSD detector by itself, however, can be used as a software selectable multi-array BSE detector for imaging. Such BSE microstructure images provide information that may be useful to an EBSD analysis. The EBSD pattern itself need not be indexed, therefore BSE imaging using an EBSD system is applicable to non-crystalline and bioscience materials as well. The technique is fast, convenient, and inexpensive. This article describes the system and gives some application examples.

Standard BSE imaging in the SEM. Although specimen surfaces are more commonly imaged in the SEM by collecting low-energy SEs, a signal derived from BSEs at energies close to that of the primary beam can also contain valuable information [1]. The number of electrons that are backscattered from a certain spot of the specimen is dependent on the local inclination of the surface, the local elemental composition, and the crystallographic orientation in the near-surface region of the specimen. These signals give rise to topographical contrast, atomic number contrast, and orientation contrast (also known as channeling contrast), respectively. Images produced with BSEs are less affected by minor specimen charging than SEs because the BSEs are of high energy.

BSE imaging with an Everhart-Thornley detector. Conventional imaging in the SEM with a scintillatorphotomultiplier detector (Everhart-Thornley type) tends to yield a higher spatial resolution, particularly when the signal collected contains significant numbers of SEs from the area under the electron beam footprint. Low-energy secondaries can reach the detector even from highly tilted specimen surfaces because a positive voltage is applied to a screen in front of the detector. SEs are mostly used for imaging topography with typical soft contrast. Atomic-number contrast and orientation contrast, however, are generally weak with an E-T detector because only a small fraction of BSEs will reach the small area detector from regions that are on a straight visual sight line to the detector.

BSE imaging with overhead detectors. Dedicated BSE detectors are usually sited just beneath the SEM objective lens directly above the specimen. Two variants are in common use [1]: (a) an annular solid-state diode detector consisting of a thin large-area silicon diode with two or four detection sections, and (b) an annular scintillator connected through a light pipe to a photo-multiplier tube (Robinson design). Each detector subtends a large solid angle and collects a substantial fraction of the electrons backscattered from a sample in a horizontal position. Because these detectors are mounted directly above the specimen, the BSE signal contains little topographical information from a horizontal specimen. Topographical contrast is further suppressed by preparing a flat polished surface. Atomic-number contrast dominates, whereas oriention contrast is usually weak.

For orientation contrast imaging, especially in polyphase rock and minerals, the sample is steeply tilted and forescatter electron (FSE) detectors are mounted below the specimen such that high-energy, elastically scattered electrons are collected at angles close to a simple reflection path. In this particular geometry, orientation contrast and atomic-number contrast signals have similar magnitudes [2].

A promising new technique is the combination of EBSD analysis and channeling contrast imaging for observing crystal defects, such as dislocations, stacking faults, twins, and grain boundaries in the SEM [3]. This technique provides Burgers vector analysis of dislocations, but this approach requires a precision goniometer sample stage in the FE SEM to enable controlled sample tilts through large angles.

BSE imaging at steep sample tilts. In EBSD measurements [4], as well as in experiments intended to render subtle topographical contrast more visible or to reduce the penetration depth of the primaries, the specimen surface is steeply tilted, typically to a plane $70^{\circ}$ above the horizontal. When specimens are tilted to high angles, standard BSE detectors on the ceiling of the specimen chamber (Figure 1, location a) collect few electrons because of strong forwardscattering of BSEs. So the overall number of BSEs captured is too low to be useful: the weak signal results in a noisy image.

To enable imaging in the highly tilted position, either the EBSD detector must be retracted and a special BSE detector moved into a suitable position or small solid-state semiconductor detectors must be mounted on the edges of the EBSD phosphor screen (Figure 1). In the latter configuration the EBSD detector can be left in place, which is a more convenient option for commercial EBSD systems. If the BSEs are acquired with a solid-state detector at the topside of the screen (Figure 1, location b), they provide mainly atomic number contrast in the image. If the solid-state detector is placed beneath the screen (Figure 1, location d), orientation contrast images are obtained by collecting highenergy electrons that are scattered from the sample in forward direction at shallow exit angles (FSE). Solid-state detectors on the sides of the screen are used to view surface relief (Figure 1, locations $\mathrm{c}$ and $\mathrm{c}^{\prime}$ ).

Solid-state detectors mounted on the frame of the phosphor screen have several disadvantages. To avoid shadow-casting, they must be placed outside the margins of the screen. There the intensity of BSEs is low because 


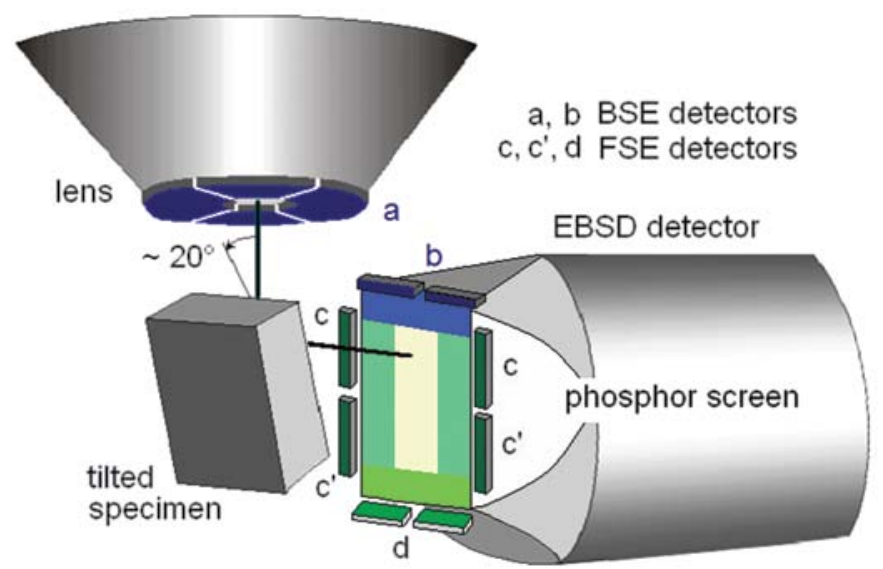

Figure 1: Schematic setup of an EBSD detector in the SEM. In addition to the physical semiconductor detectors located around the EBSD phosphor screen, software provides four acquisition boxes on the screen itself (lighter colors) that relate to the collection of BSEs and FSEs.

the EBSD detector is positioned such that the maximum of intensity is close to the screen center. The space between the specimen and the EBSD phosphor screen is quite small, typically between 1 and $2 \mathrm{~cm}$. Additional solid-state BSE detectors occupy valuable space inside the SEM vacuum chamber, space shared with the SEM stage, sample manipulation devices, an EDS detector, or a focused ion beam (FIB) appliance. Solid-state detectors are forced to be small in area, and thus only a small BSE signal would be obtained. Even worse, individual solid-state detectors are in fixed positions, which cannot be optimized for contrast enhancement. The setting of optimum gain and black level is sometimes a difficult task to undertake. Lastly, multiple solid-state detectors with dedicated electronics are costly.

\section{Methods and Materials}

Intensity streaming of EBSD data. In the FastEBSD system [5,6] unprocessed sequences of Kikuchi patterns are stored by streaming, raster point by raster point, like a movie from the EBSD detector onto the hard disk. A Kikuchi diffraction pattern on the screen can be regarded as a $2 \mathrm{D}$ intensity distribution of BSEs. In the following we use the term "pattern" also for intensity distributions of BSEs from non-crystalline samples and from heavily deformed crystals when no diffraction pattern is visible. The EBSD detector by itself can be used as a multi-array BSE detector [7] to replace supplementary solid-state detectors for conventional imaging. By extracting and evaluating the intensities in predefined sections of the EBSD patterns with appropriate software, the sample surface can be reproduced, again raster point by raster point, in atomic-number contrast, topographical contrast, or orientation-contrast images $[8,9]$. The microstructural images can be generated either on-line as a second task during pattern acquisition or off-line at any time from the stored EBSD pattern sequence.

In our system, four boxes are placed on the computer monitor screen, by tracking with the mouse, at deliberately selected positions and sizes on the patterns $[8,9]$. The collected intensities correlate with the BSE intensities released in the solid angles that are covered by these boxes during the scan across the sample. The signals so produced are similar to those acquired with conventional BSE detectors, if the latter would look on the sample at the same angles. Because the $x-y$ positions of the scan points are related to the running number of the patterns, images of the microstructure can be constructed. This new approach provides compositional atomic-number contrast, surface topography, and crystalorientation contrast images at a scale of some ten nanometers resolution. The phosphor screen of the EBSD detector has a threshold sensitivity of about $4 \mathrm{keV}$ thus acting as a crude electrostatic velocity analyzer that eliminates the signal from low-energy SEs and low-energy BSEs.

Image acquisition. Acquisition speed of this method is not significantly slower than intensity measurement with small solidstate detectors mounted on the screen frame in dark areas of the patterns. Up to 3,000 patterns per second can be acquired with the latest NORDIF UF EBSD detectors [10]. Construction of microstructure images from pattern sequences takes only a few minutes. EDAX-TLS has developed a similar approach names PRIASR [11]. They use up to 25 acquisition boxes of fixed size and position and do not use pattern streaming yet.

In the present system, each of the detector boxes captures a part of the signal in the pattern. The boxes are independent from each other and may overlap. The digital format of the raw data facilitates image processing. The signal of each box can be used individually or mixed with those from the other boxes. As with conventional analog solid-state detectors in the SEM, the signals of two boxes can be added or subtracted with adjustable weights. In addition, complex arithmetic functions can be applied. This way of processing the images by software during construction corresponds to the settings of gain and black level in conventional analog image detectors. Color-coding and mixing the individual detector signals is intended to render low contrast more easily perceptible $[8,9]$, which often helps to distinguish subtle microstructure details invisible in gray-tone images.

Quantitative mapping. EBSD analysis provides several quantitative microstructural mappings by representing, for example, the spatial distributions of crystallographic directions (Miller maps), crystallographic orientations (Euler maps), pattern quality $(\mathrm{PQ})$ magnitudes, grain misorientations, and grain boundaries [12]. A crystal orientation is defined by the rotation to bring the crystal coordinate system in coincidence with a reference coordinate frame of the sample. The rotation is expressed mathematically by a matrix, for instance, by using three Euler angles or by using three Miller indices, $(h k l)$, of the surface plane, and of three indices, $\left[\begin{array}{lll}u & v\end{array}\right]$, of a reference direction in this plane. The three Euler angle parameters may be assigned to three basic colors, red, green, and blue. For a graphical representation, each grain in a micrograph of the specimen surface may hence be stained by a unique color, which, on the other hand, can be interpreted in terms of orientation parameters by comparison with a color legend. Thus a quantitative orientation image of the sample is obtained, known as Euler map. Likewise images of the spatial distribution of crystallographic lattice planes and directions can be constructed by ascribing specific colors to the Miller indices, $(h k l)$, respectively to the direction indices, $(u v w)$. For this purpose the stereographic 
standard triangle is overlaid with a color triangle, whereby the corners are given the basic colors red for $\left(\begin{array}{lll}0 & 0 & 1\end{array}\right)$, green for ( $\left.\begin{array}{lll}0 & 1 & 1\end{array}\right)$, and blue for ( $\left.\begin{array}{lll}1 & 1 & 1\end{array}\right)$. These distribution mappings of crystallographic planes or directions are named Miller maps or, somehow confusing, inverse polefigure maps (IPF). $\mathrm{PQ}$ is a measure of the sharpness of bands in the Kikuchi pattern. It gives a qualitative indication of the surface state and the perfection of the crystal lattice. PQ maps are routinely constructed during EBSD analysis. The brighter that PQ is plotted in the map, the more perfect is the crystal under the beam.

In EBSD acquisition it is common to scan the sample from bottom to top in order to reduce contamination effects. However, the images on the monitor screen are displayed, starting with the first line, from top to bottom. That means that the image is vertically mirrored as compared to the scan field on the sample. Therefore, the boxes for acquisition of FSE are close to the top of the image, and the boxes for BSE are at the bottom area.

\section{Results}

Nickel grains. The SE image (Figure $2 \mathrm{a}$ ) of a nickel sample tilted at $70^{\circ}$ out of horizontal suffers from low intensity and contrast. Some cusps give rise to bright spots in the image. Topography at grain boundaries is faintly indicated. As opposed to this, the topographical contrast image (Figure 2d), constructed from the intensities forward-scattered in box $\mathrm{D}$, reveals an intuitively accessible relief. The raster scan was $386 \times 364$ steps. It is noteworthy that the SE image of Figure 2a, taken with a standard Everhart-Thornley detector, and the PQ map, Figure $2 b$, show a strong sensitivity to contamination. On the left side is a brighter strip in Figures $2 a$ and $2 b$. This is the clean surface. The darker field is already contaminated by a previous scan. EBSD (Figure 2c), topographical contrast (Figure 2d), and orientation contrast (Figures $2 \mathrm{e}$ and $2 \mathrm{f}$ ) imaging are not yet affected by the contamination layer from a single scan.

Dual-phase steel. Figure $3 \mathrm{a}$ shows the surface of a dual-phase steel sample containing $55.1 \%$ bcc (ferritic) and $41.5 \%$ fcc (austenite) phases according to EBSD analysis (see insert). Only $1.8 \%$ of the sampled points could be assigned likewise to fcc and bcc, and $1.6 \%$ failed to index. The latter are marked by black pixels. The raster scan was $200 \times 150$ sampled points, and the pattern sequence was stored on the hard disk. Figure $3 \mathrm{~b}$ presents an $(h k l)$ Miller plot of the sample surface. After indexing a pattern, the Miller indices of the crystal plane parallel with the sample surface are calculated, finally a color is assigned according to the location in the colored cubic standard triangle (insert top right), and the pixel is plotted. This is done for each of the other sampled points. The Miller indices can be recovered in reverse by comparison of the pixel color with that color on the triangle. Because both phases belong to the cubic crystal system, they are plotted here in one Miller map. It is also common to plot the Miller indices directly in the standard triangle to construct an inverse pole figure (see insert). Figure $3 c$ shows a microstructure image of the sample surface in false colors by an overlay of the BSE intensities from the boxes $\mathrm{A}, \mathrm{B}$, and $\mathrm{C}$, whereby the colors, red for the intensity magnitude in box A, green for the intensity magnitude in box $\mathrm{B}$, and blue for the intensity magnitude in box $C$, are attributed and mixed. It must be emphasized that these colors are not correlated with the grain orientations, in contrast to Figure $3 \mathrm{~b}$. The insert shows the positions of the acquisition boxes on the screen.
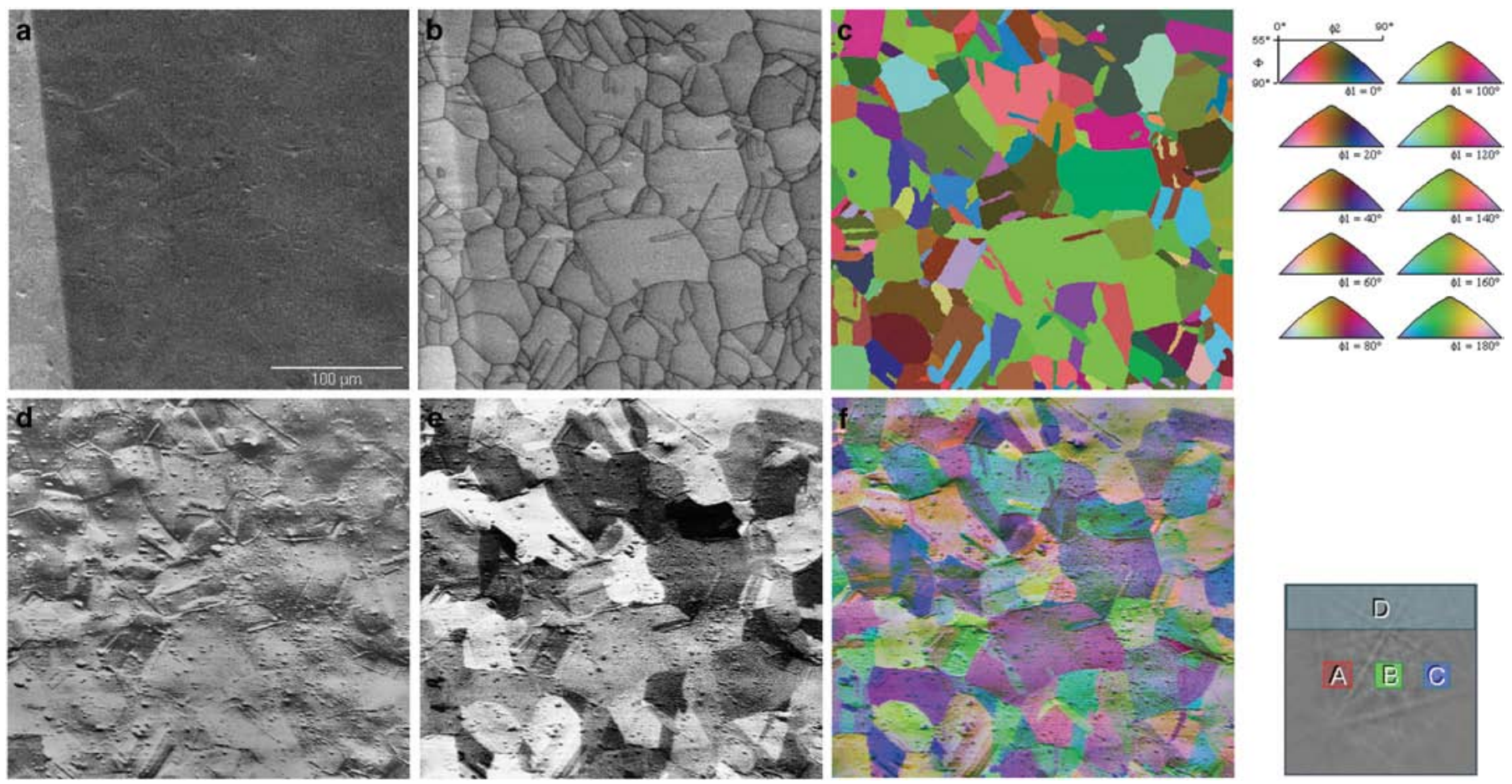

Figure 2: A recrystallized nickel surface at $70^{\circ}$ tilt out of the horizontal. (a) An SE image acquired with an E-T detector. (b) A PQ map. (c) An Euler map from EBSD analysis. (d) A topographical contrast image constructed with FSE intensities in box D. (e) An orientation contrast image constructed with BSE intensities in box B. (f) The orientation contrast image in false colors by superposition of RGB-encoded BSE intensities from boxes A, B, and C. A color legend for Euler angles is at the top-right. 

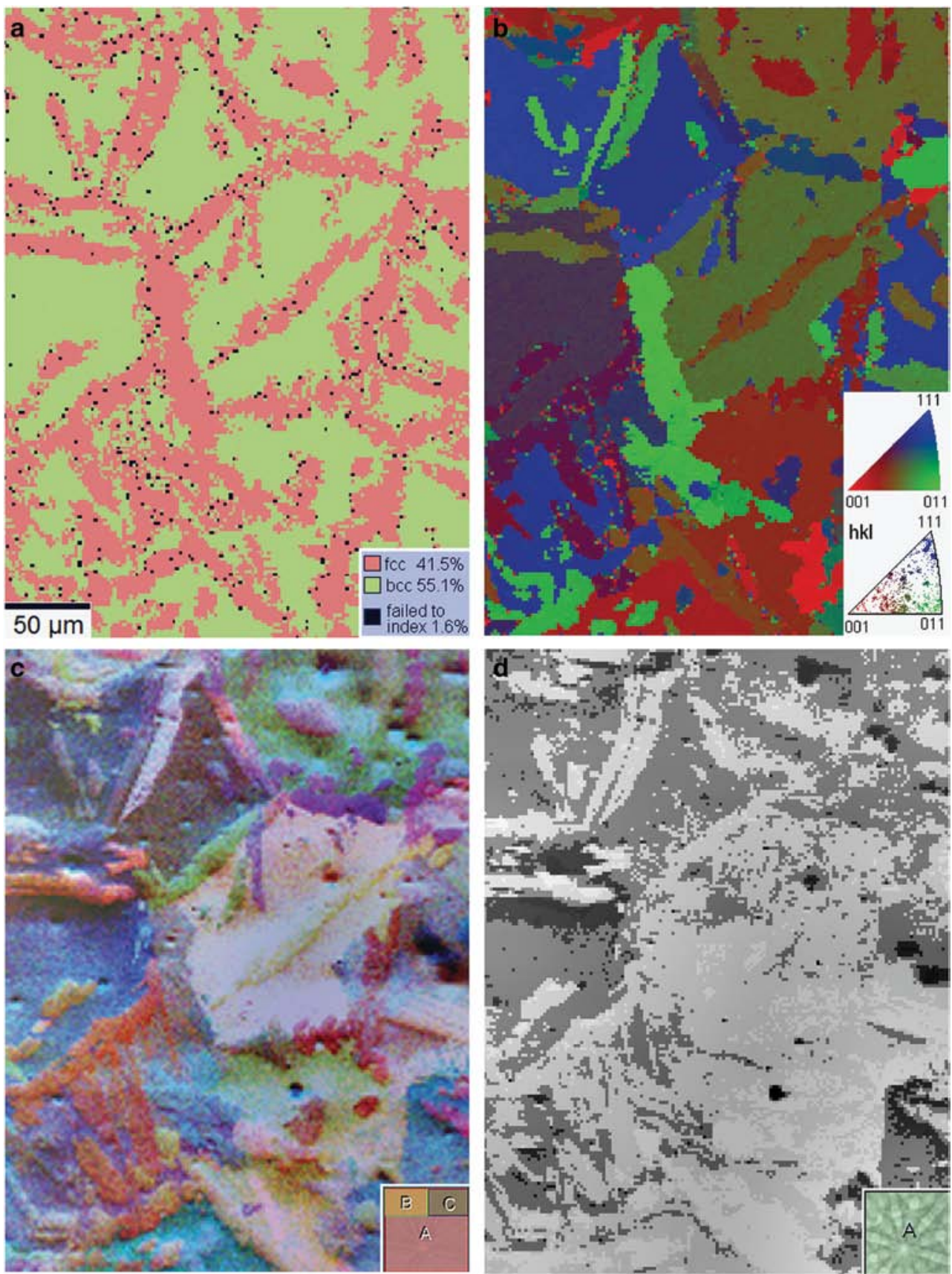

Figure 3: Dual-phase steel sample at $70^{\circ}$ sample tilt. (a) shows the distribution of the fcc and bcc phases from EBSD analysis. (b) An ( $\mathrm{k} \mathrm{kl)} \mathrm{Miller} \mathrm{map} \mathrm{of} \mathrm{the} \mathrm{sample} \mathrm{surface.} \mathrm{(c)} \mathrm{shows} \mathrm{a} \mathrm{false} \mathrm{color} \mathrm{image} \mathrm{composed} \mathrm{and} \mathrm{RGB}$ color-coded from intensities in boxes A, B, and C. (d) The surface image constructed from BSE intensities in a screen-wide acquisition box.

Figure $3 \mathrm{~d}$ is the contrast image constructed from the BSE intensities collected in a wide box of screen size. The intensity magnitudes are plotted in gray scale. The BSE yield is not only a function of the mean atomic number, the inclination of the surface, and the crystallographic orientation, but it also depends on the local condition of the sample surface. Thus, contrast may be reduced by a contamination layer or increased by a phasespecific reaction layer. All these contributions superimpose in the BSE image. In the dual-phase steel the difference in mean atomic number between the phases is small so that orientation and topographical contrasts stand out. The gray value distributions in Figure 3d, for instance, give only a first indication of the phase distribution in the sample surface. It must be verified and quantified by means of an EBSD analysis on selected points or by comparison with an EBSD phase distribution map (Figure 3a). EBSD analysis, however, presupposes a flat polished sample surface and low plastic deformation, which is not always easy to obtain, in particular with multi-phase materials. It is worth noting that EBSD does not discriminate between cubic crystals of the same Laue group or similar lattices. Therefore the gray shades in the BSE image (Figure 3d) may be an indication of some bainite or martensite content, in addition to the ferrite (bcc) and austenite (fcc) phases.

Revealing surface topography. A light optical analogy may help to intuitively interpret topographical contrast. Imagine the sample viewed along the primary beam direction and, at the same time, illuminated from the side under a spherical angle defined by the size and position of the acquisition box (Figures $4 \mathrm{~b}-4 \mathrm{~d}$ ). Thus a small box results in a contrasty image. The smaller the box and the more oblique the illumination, the stronger are the shadows. The height of elevations over a flat surface can be calculated from the position of the box and the breadth of shadows. A large box, in the extreme the size of the whole EBSD phosphor screen, leads to an image with reduced shadows (Figure 4a).

Combining images. Details in the sample surface can be further accentuated by digital image processing, such as by subtracting or adding, pixel by pixel, the intensities of one box to that of another one (Figure 4e, Figures $5 \mathrm{e}$ and 5f, Figure 6b), by color coding (Figure 3c, Figure 4f), adjusting brightness, or enhancing gamma contrast. Unusual and unrealistic contrasts, however, can arise in the combination or subtraction of these images. A major advantage of off-line evaluation of stored pattern sequences and post processing is that repeated sessions on the SEM are avoided. The user can adjust the acquisition boxes and vary contrast settings until sample details of interest are optimally seen.

Non-crystalline and non-conductive specimens. The pattern sequences of Figures 2 through 5 were acquired at $20 \mathrm{kV}$ accelerating voltage, and the pattern sequence of 


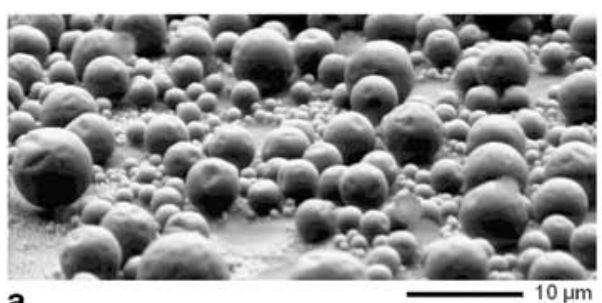

a

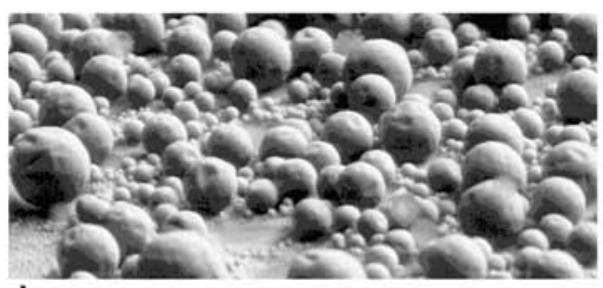

d

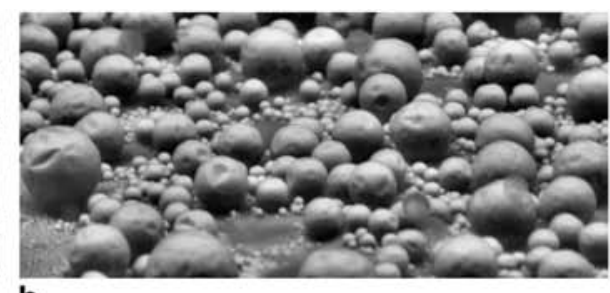

b

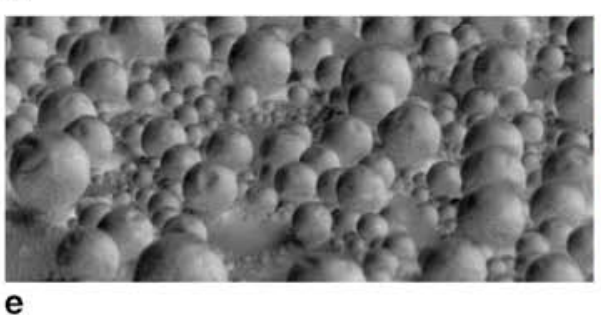

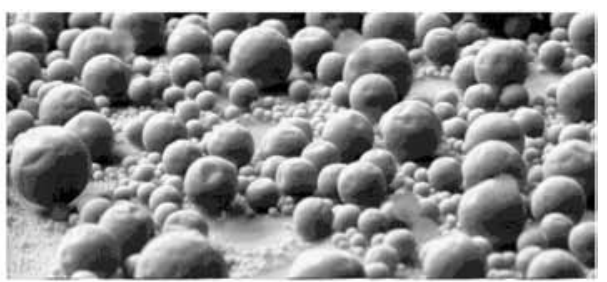

C

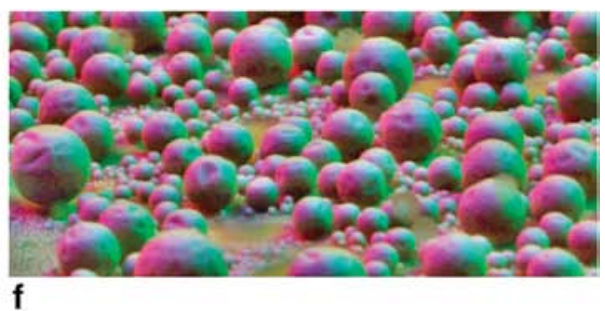

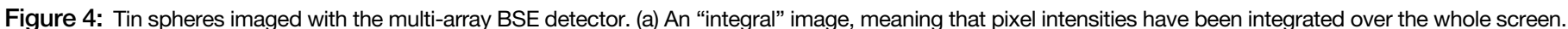
(b) Intensities have been acquired in a small box in the FSE. (c) A small box was positioned along the left margin of the screen. (d) A small box positioned along the right. (e) Pixel intensities of the right box subtracted from pixel intensities of the left box. ( $f$ A false color representation by attributing red, green, and blue to the pixels of boxes of B, C, and D, respectively. (Sample was provided by PHILIPS Eindhoven, now FEI.)

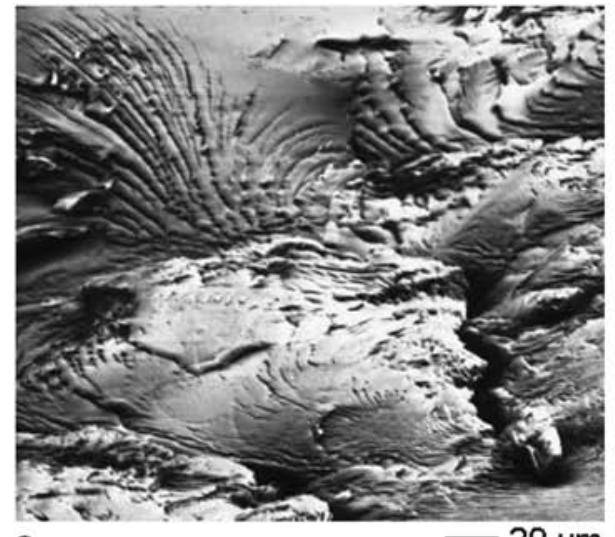

a

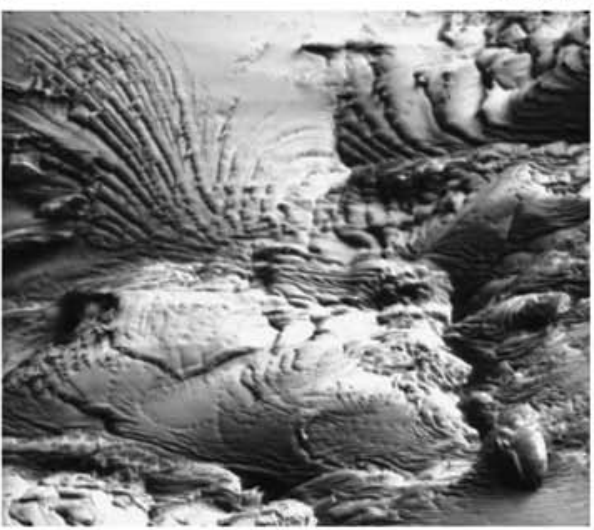

d

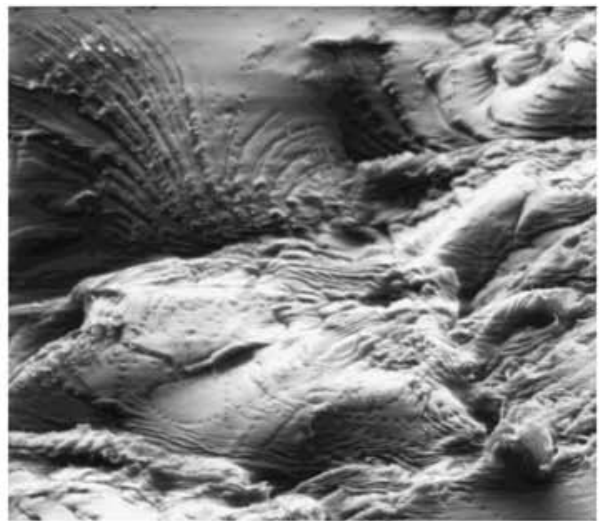

b

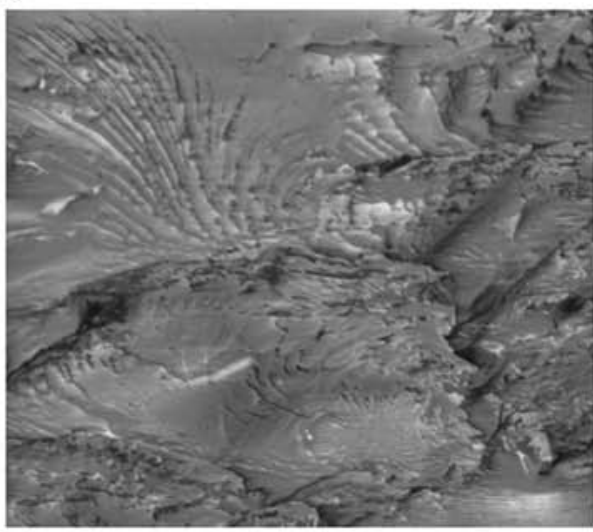

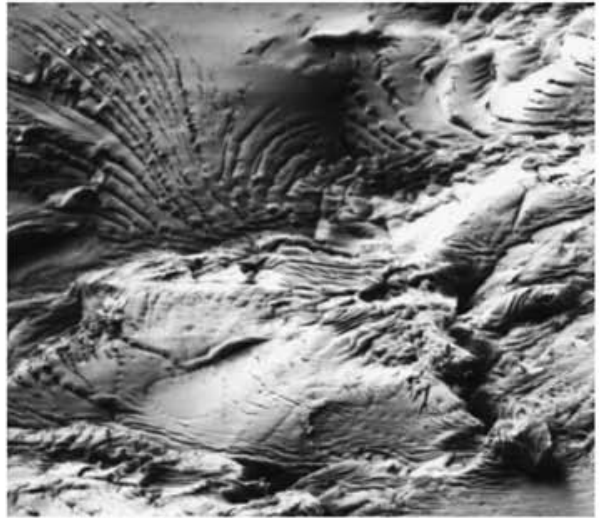

C

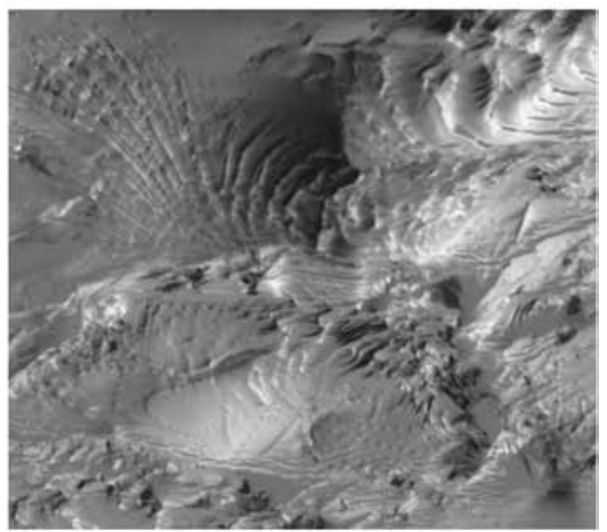

f

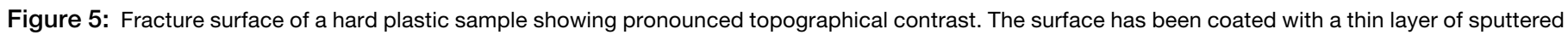

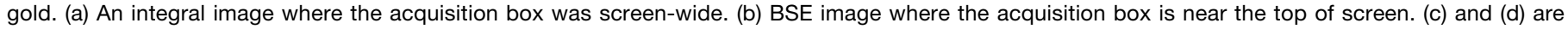

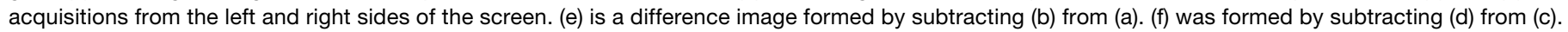

Figure 6 was acquired at $10 \mathrm{kV}$. As distinct from the construction of crystal orientation maps in EBSD analysis of crystalline samples, pattern sequences need not be indexed [7-9] for BSE imaging. Therefore, BSE imaging by using the EBSD system is applicable to non-crystalline materials (Figures 5 and 6). In addition, non-conductive specimens may be sputter-coated, as is usual in SEM work, to avoid specimen charging and to reduce radiation damage to sensitive organic materials. Grain size in sputtered metal layers is typically smaller than the normal BSE image resolution, and so they are only resolved in high-resolution imaging. 

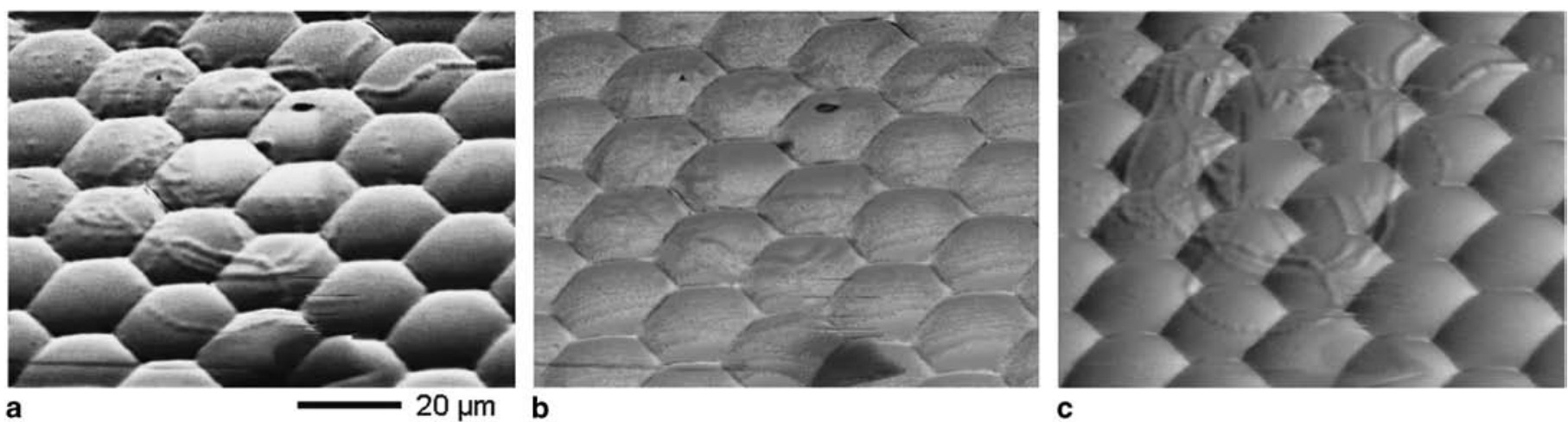

c

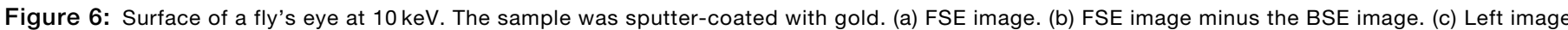

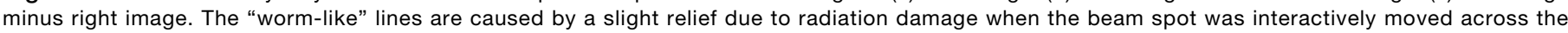
sample.

\section{Discussion}

The advantages of BSE imaging by manipulating pattern streams are a high topographical contrast and, if applicable, atomic-number contrast in the images, depending on the sizes and positions of the acquisition boxes in the patterns. The acquisition boxes can be (re)adjusted on the patterns at any time to optimize contrast, even with a computer not connected with the microscope.

The advantages of a multi-array EBSD detector over special semiconductor detectors are for imaging in an EBSD setup: (1) no restriction on the free space in front of the sample; (2) superposable alignment of images to EBSD orientation maps; (3) flexible adjustment of image contrast by selecting the acquisition boxes and digital imaging; (4) pattern sequences need not be indexed. Therefore BSE imaging by using the EBSD system applies as well to partially crystalline, non-crystalline, and bioscience materials; and (5) there are no additional hardware costs.

\section{Conclusion}

If the SEM sample is tilted to high angles, as is for instance the case in EBSD measurements, the usual BSE detectors above the sample are ineffective. Thus, special backscatter or forward scatter semiconductor diode detectors are occasionally employed, mounted on the margins of the EBSD phosphor screen. This article shows, however, that the EBSD detector by itself can be used as a BSE detector for conventional imaging. With appropriate software the sample surface can be imaged in atomic number $(Z)$, topographical, and orientation contrast. Analysis can be performed both on-line during acquisition or preferably off-line at any time by evaluating stored pattern sequences.

\section{References}

[1] RF Egerton, Physical Principles of Electron Microscopy An Introduction to TEM, SEM, and AEM. Chapter 5.4, Springer Science+Business Media Inc., New York, 2005.

[2] DJ Prior et al., Mineral Mag 60 (1996) 859-69.

[3] S Zaefferer and N-N Elhami, Acta Mater 75 (2014) 20-50.

[4] AJ Schwartz et al. (eds.), Electron Backscatter Diffraction in Materials Science, $2^{\text {nd }}$ edition, Springer Sciences+Business Media Inc, New York, 2009.

[5] RA Schwarzer and J Hjelen, Solid State Phenom 160 (2010) 295-300.
[6] RA Schwarzer and J Hjelen, Mater Sci Tech Ser 26 (2010) 646-49.

[7] X Tao and A Eades, Microscopy Today 11 (2003) $36,38$.

[8] R Schwarzer et al., "Imaging of topography and phase distributions with an EBSD detector in the SEM," Microscopy Conference Kiel 2011, Poster LBP M.P007, www.ebsd.info.

[9] R Schwarzer, Practical Metallography 51 (2014) 160-79.

[10] http://www.nordif.com

[11] SI Wright et al., Ultramicroscopy 148 (2015) 132-45.

[12] D Gerth and RA Schwarzer, Textures Microstruct 21 (1993) 177-93.

\section{Amptek FAST SDD ${ }^{\circledR}$ for EDS (SEM) Applications}

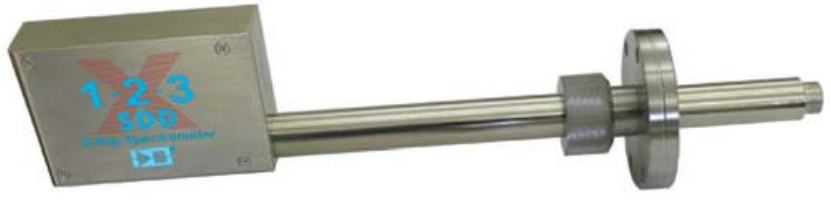

Amptek's FAST SDD ${ }^{\circledR}$ detector for EDS use with SEMs utilizes new technology "C2 Series" X-ray windows (Si3N4) and has a low energy response down to Boron. With its high intrinsic efficiency the SDD is ideal for EDS XRF. See why Amptek detectors are the \#1 choice of OEMs worldwide.

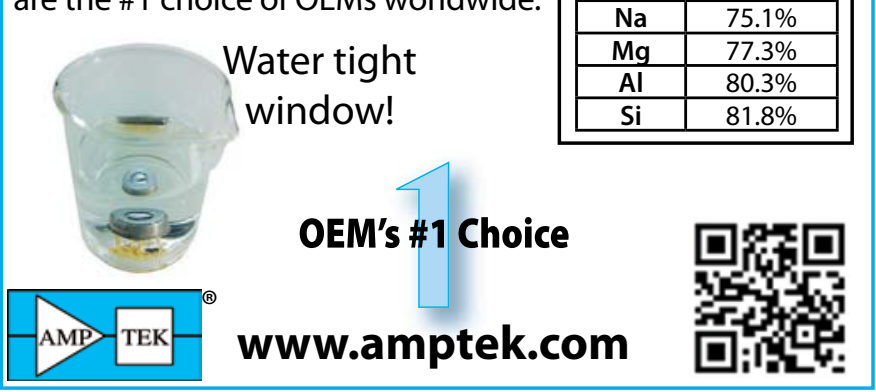

